\title{
Large and Dense LoRaWAN Deployment to Monitor Real Estate Conditions and Utilization Rate
}

\author{
Rumana Yasmin, Juha Petäjäjärvi, Konstantin Mikhaylov and Ari Pouttu \\ Centre for Wireless Communications \\ University of Oulu \\ Oulu, Finland \\ \{firstname.lastname\}@oulu.fi
}

\begin{abstract}
Internet of Things (IoT) drives today's world towards digitalization allowing diverse and innovative use cases. These use cases are changing in a fundamental way how people are conducting their business in various verticals. In this paper we focus on the real estate use case. We deploy a monitoring system in a large open space at University of Oulu. The deployed system visualizes real estate conditions of this environment and gives insight to understanding a LoRaWAN IoT-enabled building. The number of the sensor nodes composing the deployed system reaches 331, with each node comprising five sensors providing information about temperature, humidity, $\mathrm{CO}_{2}$, amount of light, and motion. In the paper we report the results and the lessons learned during the deployment of such an extensive LoRaWAN sensor network. Aside of the practicalities related to the deployment of the network, we characterize and report the performance of the deployed network. The conducted deployment can become a valuable reference for engineers and practitioners, deploying a real estate monitoring system with LoRaWAN.
\end{abstract}

Index Terms - Digitalization; Internet of Things; smart city; LoRaWAN; real estate; monitoring system; packet error rate; packet collision; RSSI; SNR.

\section{INTRODUCTION}

Digitalization and the related digital transformation, with introduction of communications between connected 'things' is a fascinating trend driving the global change. It changes human activities in many areas such as wellbeing and health, transportation, logistics, energy management, and smart building management by providing value-added tools within existing and emerging verticals and their use cases.

The Internet of Things (IoT) has a pivotal role in leveraging digital transformation and fostering innovations. With the exponential increase in the number of devices IoT can have a major impact on the different aspects of human life [1] and the way we approach the digital future. The current evolution has made IoT mature enough to enable its systematic implementation by academia and industry. To implement IoT systematically and to foster interoperability, IoT standard development organizations [2] are promoting standards for different verticals, such as, digital home management, smart industrial operations, automated traffic monitoring and control, environment pollution control, street light management, etc. IoT has already been used to monitor conditions in homes, universities, hospitals, shopping malls thus providing tools to make intelligent and digitalized applications [1]. For example, a wireless indoor monitoring system with mobile-camera-image and sensor based VLC has been implemented and tested in [3]. This system utilizes a mobile application with a camera image sensor as a light signal receiver for processing data coming from six sensor modules.

In the context of a digital campus where IoT sensors are deployed to enable smart building functionality, several use cases could be implemented [4]. For example, receiving notifications about unusual values of temperature, maintaining humidity levels for a sensitive biological laboratory, restricting $\mathrm{CO}_{2}$ level to improve the air quality, and getting intrusion alarms by monitoring unexpected movements in an area.

In this paper, we focus on building an IoT-based digital campus inside the University of Oulu by implementing an end-to-end IoT system with large and dense deployment. The installed sensor nodes keep track of temperature, humidity, $\mathrm{CO}_{2}$, mobility and light intensity in a designated area. These data can be delivered, e.g., to an application running on smart phone of a university visitor. Alternatively, the data from sensor nodes can also be retrieved from anywhere using application programming interfaces (API). This makes the established real time monitoring system one of key aspects of a digital campus.

In future the functionality of the network may be enriched. For example, the network may be utilized to ensure human comfort, providing control over ventilation, air conditioning and heating systems. At the same time the deployed network is a testbed, which can be utilized to study the practicalities of the massive machine type communications in real estate use case. 


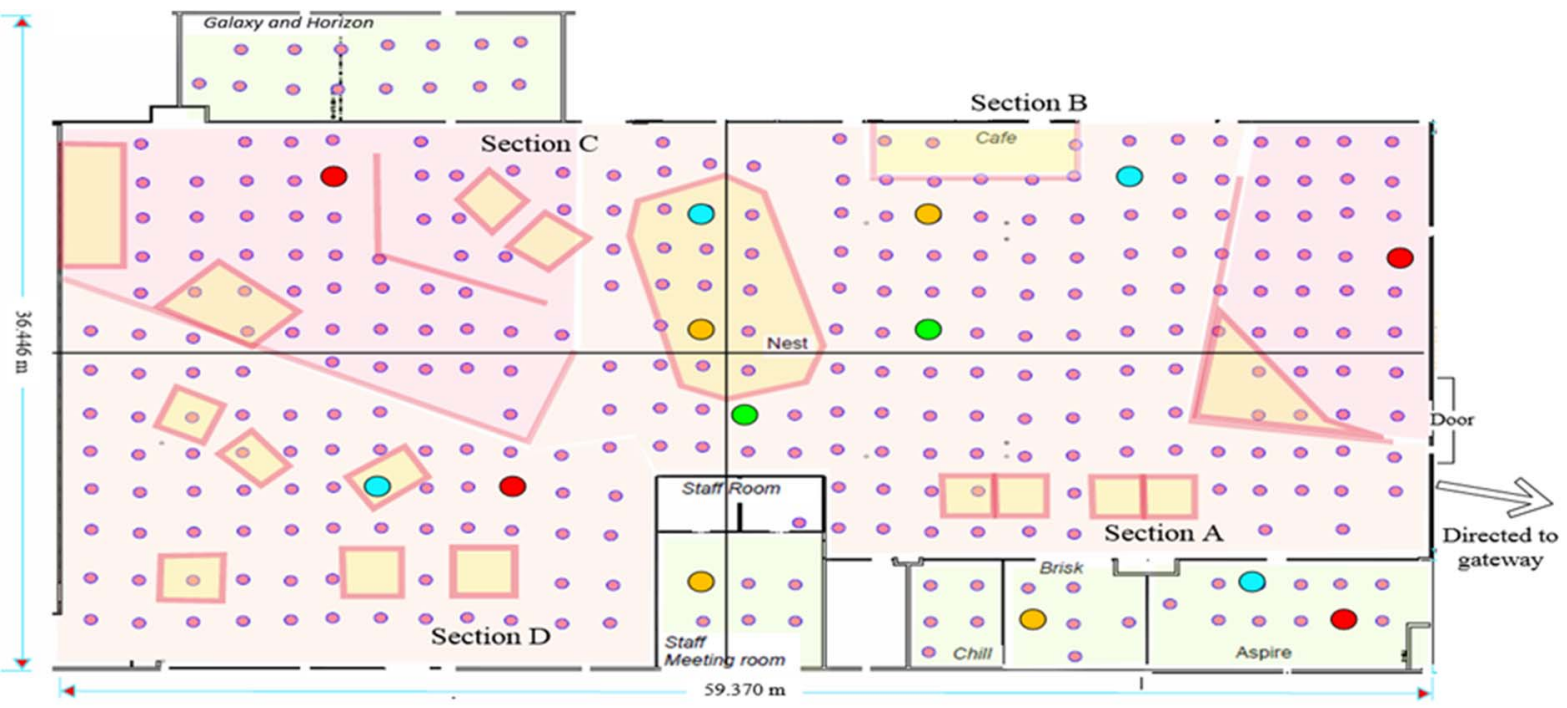

Fig. 1. Sensors deployment scenario in Tellus Innovation Area at University of Oulu.

The landscape of the available today IoT technologies is really diverse. Some IoT use cases require frequent transmissions, the other - ultrareliability and low latency. When this comes to a real estate monitoring vertical, the need of covering sufficiently large areas while keeping the capital and operation expenditures low is the major challenge. Among the available today communication technologies, the ones, which can be jointly addressed as Low Power Wide Area Network (LPWAN) seem to be the most perspective choice for such use case [4].

To this day, multiple different LPWAN technologies has been proposed, including, e.g., Sigfox [5], INGENU [6], Weightless-P [7], NB-IoT [8], and LoRaWAN [9]. Of these technologies, LoRaWAN is one of the most well-established ones. Also, unlike its counterparts, this technology enables deployment both of a public (i.e., operator controlled) and of a private (i.e., user controlled) network. The latter approach can be especially beneficial in combination with the microoperator concept [10], which implies that a real estate's owner could act as a communication infrastructure provider for its premises.

A LoRa gateway can support seamless connectivity with network server by using, e.g., a 4/5G backhaul [11], thus providing ubiquitous coverage which can have a fundamental role in the proliferation of IoT. When it comes to large-scale deployment in indoor and outdoor locations, LoRaWAN is rather mature in availability and it is straightforward to deploy [12].

The paper starts with the motivation and reasons behind the development towards a digital campus, which enables real estate use cases. The LoRa sensors deployment at the Tellus Innovation Area inside University of Oulu is described and discussed. This gives a practical insight into the possible use cases and challenges faced in IoT deployments such as methodology, complexity, and limitations. After the deployment, a measurement campaign is carried out to gain a further understanding of the IoT-enabler technologies, communications networks, sensors and the required cloud infrastructure. We then discuss the performance of the deployed sensor network in terms of packet error rate (PER), signal-to-noise ratio (SNR) and received signal strength indicator (RSSI). Finally, we conclude by summarizing the obtained results.

\section{REAL EstATE MONITORING SYSTEM}

Based on the digital campus concept and to realize a data monitoring platform, IoT capable sensor test bed is deployed at a crowded location within the University of Oulu premises. The location for the large-scale sensor deployment is the Tellus Innovation Area [13]. This area, approximately 2163.8 square meter (depicted in Fig. 1), is usually crowded with students and staff members during. This location is interesting because it is an open space designed for brainstorming, exploring diverse cultures from inside and outside of the university, facilitating activities related to university-company collaboration, entrepreneurship and international affairs.

The environmental parameters of interest are temperature, light intensity, $\mathrm{CO} 2$, and humidity. In addition, we wanted to be able to track people's movements so passive infrared (PIR) motion sensor was introduced as well. The PIR sensor is able to detect motion within $2 \mathrm{~m}$ range approximately. In total, 331 sensor nodes were deployed to cover the whole Tellus Area. Each sensor node unit has the listed above five sensors. Typically, temperature, $\mathrm{CO}_{2}$, and humidity do 
not change rapidly, thus packet transmission is scheduled after every 900 seconds interval.

\section{A. LoRa Wide Area Network}

The LoRaWAN technology implements ALOHAtype media access protocol for transmitting information from sensor nodes to a gateway. The interesting technical features of this technology include high tolerance to frequency mismatch, and secure communication with customized adaptive data rate (ADR) achieved through the LoRa modulation technique. According to LoRa specification [8], the duration of packet on air can be calculated while payload comprises of 8 bytes preamble symbol, synchronization word, additional cyclic redundancy check bit (CRC) and 4/5 coding rate, as [14]:

$$
\begin{array}{r}
T_{O A}=\left(\frac{2^{S F} \times 12.25}{B W}\right)+\{8+ \\
\left.\max \left(\operatorname{ceil}\left(\frac{8 P L-4 S F+28+16 C R C-20 I H}{4(S F-2 D E)}\right)(C R+4), 0\right)\right\} .
\end{array}
$$

where $D E$ indicates the low data rate optimization which could either be enabled by using 1 or be disable using 0 , $\mathrm{CRC}=1$ and $\mathrm{IH}=0$. In Europe, LoRaWAN typically uses the frequency band of 863-870 MHz. This band is unlicensed and since LoRaWAN does not utilize a listenbefore-talk, it is bounded by duty cycle restrictions. Namely, the next transmission in a sub-band is restricted for certain time period. Meanwhile, the other sub-bands can be utilized. By the means of modifying the spreading factor (SF), which affects to the data rate (DR) directly, the on-air transmission time can be traded for the range.

\section{B. Deployment methodology}

The methodology of practical LoRaWAN deployment is described as a flow of activities that we followed during our large-scale deployment. First, keeping in mind the coverage area of sensor nodes and the area of the target area, we calculated the total required number of sensor nodes for that location. Second, we have analyzed the feasibility of using LoRaWAN technology and specified the network parameters. Third, we selected the position of the GW to provide a good coverage over the target area. Fourth, we have conducted some practical tests to characterize the quality of signal at different DR from area of deployment and selected the DR to be used by our sensor nodes. Having completed the deployment, we did network tests to ensure the whole setup is working properly.

\section{Pre-deployment analysis}

Consider a network having $M$ sensors connected to a single gateway. A packet of $T_{O A}$ milliseconds long is transmitted by each sensor node periodically, using the same SF. One of the three channels is selected randomly for transmission.

We also imply following for simplicity:

- Each packet has constant length.
- No interference from external networks.

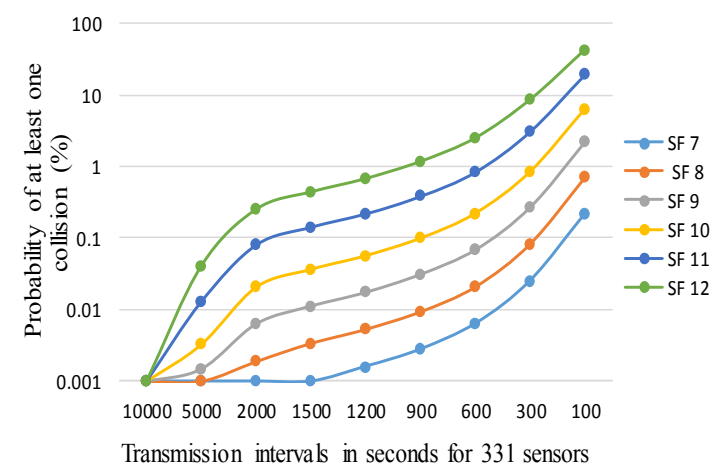

Fig. 2. Effect of the SF and report period on the probability of collisions for 331 sensor nodes ( 24 byte payload, 3 channels, one packet per interval, calculated using (2))

- Period between sequential transmissions exceeds the duration of transmission back-off.

- No acknowledgements, adaptive data rate or downlink is present.

- The initialization time for each node is random.

Since, transmissions are random, a collision occurs if two or more nodes attempt to transmit at the same time. With $T_{O A}$ calculated from Equation (1), the expected number of packets generated within certain time frame duration (following Poisson distribution [15]), is calculated by multiplying time-on-air for each packet with arrival rate, $\lambda$, which equals $M / T C$ for our case. The probability of collision can now be formulated as,

$$
P_{k}\left(T_{O A}\right)=1-\left(\sum_{k=0}^{1} \frac{\left(\lambda T_{O A}\right)^{k} \exp ^{-\lambda T_{O A}}}{k !}\right)
$$

where, value of $k=0$ and 1 , indicates that either no packets or only one packet is transmitted in a channel during the given period. The packet collisions occur when $k>1$. The effect of the SF and T on the probability of having at least one collision is illustrated in Fig. 2.

\section{Network deployment and configurations}

First, we deployed the gateway. The Multitech Conduit gateway, which can operate over a $4 / 5 \mathrm{G}$ cellular backhaul, has been used. To provide a good coverage, the gateway's antenna was placed on the University of Oulu antenna tower at a height of approximately $24 \mathrm{~m}$ from the ground-level. The distance from the gateway to the nearest point of the target deployment area is approximately $180 \mathrm{~m}$. The gateway was configured to use the default frequency plan, including the three default LoRaWAN channels. A special application has been deployed on top of the gateway to forward the collected sensor data to the ThingWorx IoT platform [16], which stored all the collected data, over MQTT protocol (for further details refer to [11]). 


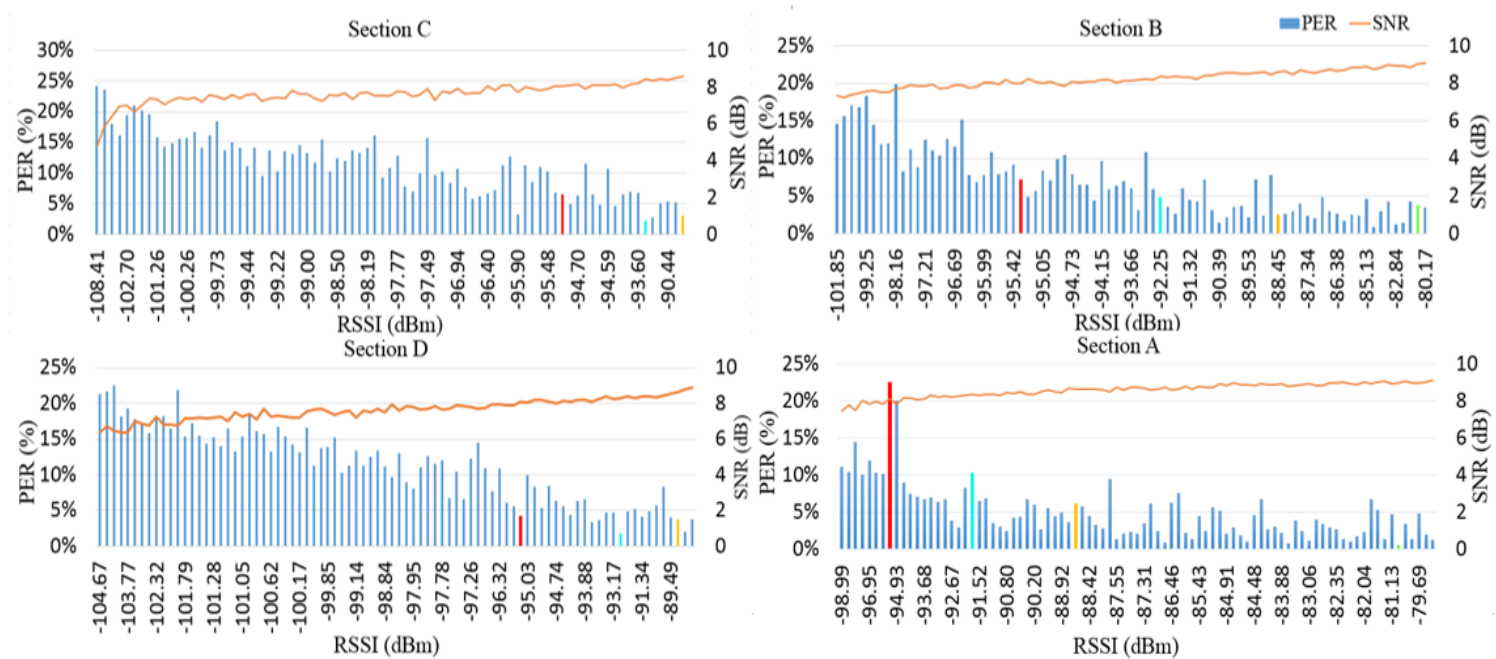

Fig. 3. Packet error rate of the deployed 331 sensor nodes (DR5 (SF7), packet payload 24 bytes, transmission duration 900 seconds and three frequency channel).

Using few test devices, we characterized the performance of the communication for the different $\mathrm{SF} / \mathrm{DR}$ between a test device located in the various points in the test area. The tests have shown that from any point in the target area a sensor node can reach the gateway using any SF/DR. For this reason we decided to use in our network the minimum SF possible, i.e. SF7. The use of SF7 on one hand minimizes the energy consumption, and on the other hand - minimizes the probability of collisions. Since the sensor nodes are static and, the ADR mechanism has been disabled. Also, since the distance between the sensor nodes and the GW is rather small, we have disabled the transmit power control.

In order to increase the lifetime of the sensor nodes and reduce the collisions probability we have set the report period of the sensor nodes to 15 minutes. Given that most of the sensed parameters are slow-changing, this setting is reasonable. Also, this results in the collision probability of below $0.1 \%$. Note that, no acknowledgement or retransmission mechanism is used.

For our deployment we selected the Elsys's LoRaWAN nodes [17]. The nodes have been configured with the parameters listed above and the parameters needed for enabling the over-the-air activation in our network, and deployed. The location of all deployed nodes is illustrated in Fig. 1. The packet flow from sensor network to application interface, technical details and challenges are available in [11].

\section{NETWORK PERFoRMANCE VALIDATION}

Once the setup was deployed, data from the sensor nodes were stored for 51 days. The total number of the received packets is 1620576 (approx.). In addition to the sensor values, such parameters as the packet sequence number, RSSI and SNR were logged. By analyzing the packets' sequence numbers we have estimated the PER.
TABLE I. Summary OF THE MeAsurement Results

\begin{tabular}{|c|c|c|c|c|c|}
\hline Packet & \multicolumn{5}{|c|}{ Measurement Area } \\
\cline { 2 - 6 } error rate & $\begin{array}{c}\text { Section } \\
\boldsymbol{A}\end{array}$ & $\begin{array}{c}\text { Section } \\
\boldsymbol{B}\end{array}$ & $\begin{array}{c}\text { Section } \\
\boldsymbol{C}\end{array}$ & $\begin{array}{c}\text { Section } \\
\boldsymbol{D}\end{array}$ & $\begin{array}{c}\text { Total } \\
\text { Area }\end{array}$ \\
\hline Mean $^{\mathbf{a}}$ & $5.03 \%$ & $6.93 \%$ & $11.17 \%$ & $11.33 \%$ & $8.56 \%$ \\
& & & & & \\
\hline Variation $^{\mathbf{b}}$ & $0.14 \%$ & $0.20 \%$ & $0.25 \%$ & $0.28 \%$ & $0.53 \%$ \\
\hline $\begin{array}{c}\text { Standard }^{\mathbf{c}} \\
\text { deviation }\end{array}$ & $3.87 \%$ & $4.48 \%$ & $5.00 \%$ & $5.29 \%$ & $7.24 \%$ \\
\hline \multicolumn{6}{|c|}{ Overall packet error rate from received packets. } \\
U. \\
Using variation formula. \\
c. Using standard deviation formula.
\end{tabular}

To facilitate the analysis, we divided the whole test area in four sections, labelled A, B, C and D (refer to Fig. 1). The data from each of these sections was processed separately. The sensors' RSSI, SNR and PER are depicted in Fig. 3. Note that, in each section we have selected three to four individual nodes. These nodes are marked with four colored markers and each marker is cross referenced in Fig. 3. For instance, the red marker in section A shown in Fig. 1 is characterized in Fig. 3 with mean RSSI and SNR value of 95.6 and 8.2, respectively.

Using data over a period of 28 days from $17^{\text {th }}$ September to $14^{\text {th }}$ October, 2017, the analysis is further narrowed down to four sensor nodes from section A. Those selected sensors, marked with four colored markers in Fig. 1, are cross referenced in Fig. 4. The SNR, RSSI and PER appears to have a random variation, so we further chose a single sensor (yellow colored) from section A and plotted all the packets from it received on two different days - when no packets were lost, and when the maximum number of packets were lost. The respective results are shown in Fig. 4 (b) and (c).

The results for the complete network and for each section are summarized in Table I. In addition to the mean values, the variation and standard deviation have been calculated and are presented as well. 


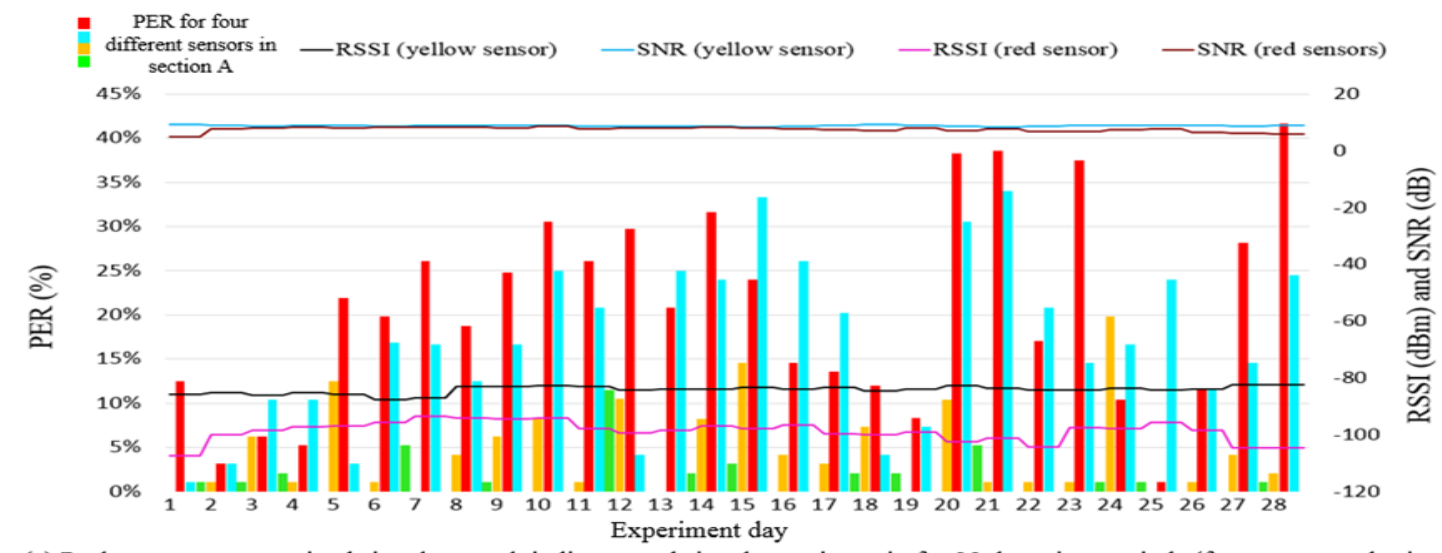

(a) Packet error rate, received signal strength indicator and signal-to-noise ratio for 28 days time periods (four sensor nodes in section A).

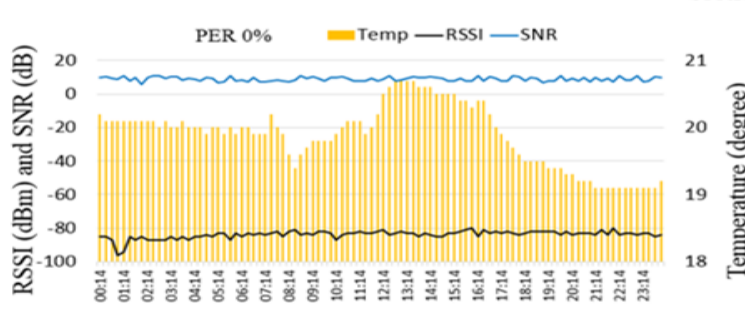

Time

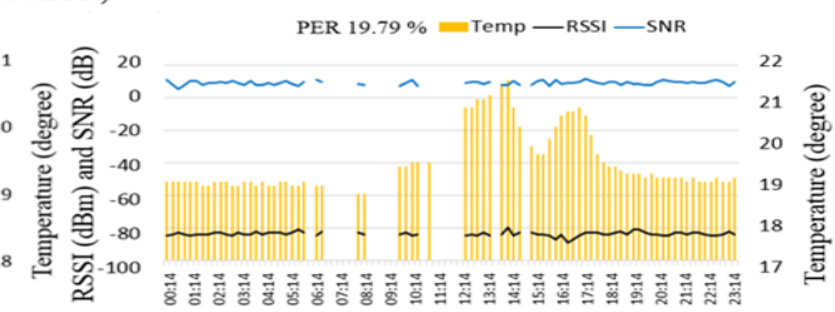

Time

b) Received signal strength indicator and signal-to-noise ratio of a (c) Received signal strength indicator and signal-to-noise ratio of sensor for a single day when packet error rate $0 \%$, also temerature a sensor for a single day when packet error rate $19.79 \%$, also level for that day. temerature level for that day.

Fig. 4. Variation of PER, RSSI and SNR in time for the selected nodes in section A and the illustrative temperature measurements for selected sensor node during the two selected days.

\section{DISCUSSIONS}

The general conclusion of the 28 day trial is that the deployed network performs reasonably well for implementing an IoT-enabled smart real estate use case. As an illustration, the temperature reports of a single sensor for a single day timeframe are depicted in Figs. 4 (b) and (c). The other parameters, namely the humidity, $\mathrm{CO}_{2}$, light intensity and motion have been reported similarly. The presented results show that the data has been received from all 331 nodes through all the days and none of the sensors had more than $25 \%$ of their packets lost. At the very same time, for some of the nodes less than $0.5 \%$ of packets has been lost.

As one can see from Fig. 3 and Table 1, the RSSI and SNR values for the nodes in Sections A and B are higher in comparison to that of nodes in sections $\mathrm{C}$ and $\mathrm{D}$. This can be explained by the fact that sections $\mathrm{A}$ and $\mathrm{B}$ are closer to the gateway. Therefore they are likely to have a better channel and may get their packets received even under the interferences from nodes in sections $\mathrm{C}$ and $\mathrm{D}$ due to the capture effect [18]. Not surprisingly, the PER in sections $\mathrm{C}$ and $\mathrm{D}$ is also on average higher than in sections A and B. Even though, the experienced levels of
PER are seemed to be excessively high, if compared to the results of the analysis depicted in Fig. 2.

To address this issue, we have selected several nodes and studied how their SNR, RSSI and PER varied in time. The presented results show that the SNR and RSSI values for these sensors do not change significantly in time. Among the reasons which may have caused such behavior are: malfunction of the devices (e.g., the gateway), the interferences from external systems, or a temporal shadowing of a node by an obstacle affecting radio wave propagation. Another possibility are the packet losses between the gateway and the ThingWorx IoT platform. In our case the gateway was connected to the Internet over Ethernet and the packets were delivered to ThingWorx via a third-party MQTT broker. Therefore, the losses were possible both between the gateway and the broker, and between the broker and the IoT platform. We plan to continue the processing of the collected data, applying advanced statistical data processing methods, to figure out the reason after the high PER.

\section{CONCLUSIONS}

The process of digitalization changes the face of the modern world. The new use cases across the various verticals, such as the automotive industry, intelligent 
transportation systems and smart home appear every single day. The real estate control systems is not an exception and illustrates well the new utility enabled by the IoT. For example, a smart lighting system can reduce energy loss by switching the excessive lights off. An air conditioning and ventilation system can reduce air pollution and maintain air quality. The continuous monitoring and controlling capability of IoT-enabled buildings can preempt an inappropriate occurrence even before being noticed by human. Real-time monitoring can enhance internal security. External information such as weather and outdoor environmental conditions can be utilized for increasing the performance even further.

This paper presents an IoT-enabled digital infrastructure forming such a digitalized ecosystem. Several steps have been taken during this deployment. First, we have formulated the methodology of a system's deployment. Second, following the proposed methodology, we analyzed the feasibility of the targeted application and selected the relevant configuration parameters. Third, we have deployed at University premises the test network composed of 331 sensors nodes, each having five different sensor, monitoring temperature, humidity, $\mathrm{CO} 2$ level, light intensity and motion. Each of these sensors streams every 900 seconds its data to the ThingWorx IoT platform, from where the data can be accessed anytime and anywhere. Finally, the data collected over a 28-day trial period has been analyzed and the initial results were reported.

The presented results confirm the feasibility of the taken strategy and the made design decisions. Also they confirm the possibility of deploying an extensive and dense real estate monitoring solution based on the LoRaWAN technology. Nonetheless, the low packet delivery rate (of only $75-80 \%$ for some nodes) was rather surprising for us. In future we plan to focus specifically on this issue and try to identify the reasons for such behavior. Also we plan to continue our experiments and measurements within the developed test bed, study the means to visualize such massive data in different ways, and implement different use cases on top of this platform.

\section{ACKNOWLEDGMENT}

This work has been partially funded by the Finnish Funding Agency for Innovation (Tekes) through VIRPA $\mathrm{C}$ project. The acquisition of the hardware for this study has also been partially funded by the ERDF project A71720, 'Big Data for 5G', governed by the HILLA program (www.hilla.center). This research has been financially supported by Academy of Finland 6Genesis Flagship (grant 318927).

\section{REFERENCES}

[1] E. M. Oproiu, M. Iordache, C. Patachia, C. Costea and I. Marghescu, "Development and implementation of a
Smart City Use Case in a 5G mobile network's operator," in Proc. 25 th Telecommun. Forum, 2017, pp. 1-4.

[2] ETSI, "SmartM2M; IoT Standards landscape and future evolutions," 2016. [Online]. Available: http://www.etsi.org/deliver/etsi_tr/103300_103399/103/ 01.01.01_60/tr_103375v010101p.pdf.

[3] Z. Ong, V. P. Rachim and W. Y. Chung, "Novel Electromagnetic-Interference-Free Indoor Environment Monitoring System by Mobile Camera-Image-SensorBased VLC," IEEE Photonics J., vol. 9, pp. 1-11, Oct. 2017.

[4] M. Loriot, A. Aljer and I. Shahrour, "Analysis of the use of LoRaWan technology in a large-scale smart city demonstrator," in Proc. Int. Conf. Sensor Netw. Smart Emerg. Techn., 2017, pp. 1-4.

[5] Sigfox, 2018, [Accessed: 2018-02-10]. [Online]. Available: http://www.sigfox.com/en/.

[6] Ingenu, 2018, [Accessed: 2018-02-12]. [Online]. Available: https://www.ingenu.com/.

[7] Weightless, 2018, [Accessed: 2018-02-12]. [Online]. Available: http://www.weightless.org/.

[8] A. S. Petrenko, S. A. Petrenko, K. A. Makoveichuk and P. V. Chetyrbok, "The IIoT/IoT device control model based on narrow-band IoT (NB-IoT)," in Proc. IEEE Conf. Russian Young Researchers Elect. Electron. Eng., 2018, pp. 950-953.

[9] LoRa alliance, 2018, [Accessed: 2018-01-09]. [Online]. Available: https://www.lora-alliance.org.

[10] M. Matinmikko, M. Latva-aho, P. Ahokangas and V. Seppänen: "On regulations for 5G: Micro licensing for locally operated networks", Elsevier Telecommun. Policy Mag., pp. 1-14, 2017.

[11] R. Yasmin, J. Petäjäjärvi, K. Mikhaylov and A. Pouttu, "On the Integration of LoRaWAN with the 5G Test Network", in Proc. 28th Annu. Int. Symp. Personal, Indoor Mobile Radio Commun., 2017, pp. 1-6.

[12] J. Petäjäjärvi, K. Mikhaylov, R. Yasmin, M. Hämäläinen and J. Iinatti, "Evaluating of LoRa LPWAN Technology for Indoor Remote Health and Wellbeing Monitoring", Int. J. Wireless Inf. Netw., vol. 24, pp. 153-162, 2017.

[13] University of Oulu, 2018, [Accessed: 2018-03-09]. [Online]. Available: http://www.oulu.fi/tellusarena.

[14] Semtech, "SX1272/3/6/7/8: LoRa Modem Designer's Guidde”, July 2013.

[15] A. S. Tanenbaum and D. J. Wetherall, "The medium access control sublayer," in Computer networks, $5^{\text {th }}$ ed. Boston, MA, USA: Pearson Education, Inc., 2011.

[16] Thingworx, 2018, [Accessed: 2018-01-09]. [Online]. Available: https://www.thingworx.com/.

[17] Elsys, 2018, [Accessed: 2018-03-11]. [Online]. Available: https://www.elsys.se/en/.

[18] K. Mikhaylov, J. Petäjäjärvi and J. Janhunen, "On LoRaWAN Scalability: Empyrical Evaluation of Technology Succeptability to In Network Interference", in Proc. Eur. Conf. Netw. Commun., 2017, pp. 1-6. 